\title{
The Cahn-Hilliard equation with elasticity_finite element approximation and qualitative studies
}

\author{
HARALD GARCKe, MARTIN RUMPF AND ULRICH WEIKARD ${ }^{\dagger}$ \\ Universität Bonn, Institut für Angewandte Mathematik, Wegelerstr. 6, 53115 Bonn, Germany
}

[Received 3 December 1999 and in revised form 2 August 2000]

\begin{abstract}
We consider the Cahn-Hilliard equation-a fourth-order, nonlinear parabolic diffusion equation describing phase separation of a binary alloy which is quenched below a critical temperature. The occurrence of two phases is due to a nonconvex double well free energy. The evolution initially leads to a very fine microstructure of regions with different phases which tend to become coarser at later times.

The resulting phases might have different elastic properties caused by a different lattice spacing. This effect is not reflected by the standard Cahn-Hilliard model. Here, we discuss an approach which contains anisotropic elastic stresses by coupling the expanded diffusion equation with a corresponding quasistationary linear elasticity problem for the displacements on the microstructure.

Convergence and a discrete energy decay property are stated for a finite element discretization. An appropriate timestep scheme based on the strongly A-stable $\Theta$-scheme and a spatial grid adaptation by refining and coarsening improve the algorithms efficiency significantly. Various numerical simulations outline different qualitative effects of the generalized model. Finally, a surprising stabilizing effect of the anisotropic elasticity is observed in the limit case of a vanishing fourth-order term, originally representing interfacial energy.
\end{abstract}

Keywords: Cahn-Hilliard equation; fourth order parabolic equation; finite element approximation; elasticity; adaptive methods; second order time discretization

\section{Introduction}

It is the aim of this paper to present a fast and reliable numerical method for the Cahn-Hilliard equation with elasticity. The Cahn-Hilliard model was introduced in $[4,5]$ to describe phase separation and coarsening in binary alloys. Phase separation occurs when a uniform mixture of the alloy is quenched below a certain critical temperature underneath which the uniform mixture becomes unstable. As a result, a very fine microstructure of two spatially separated phases with different concentrations develops. In later stages of the evolution on a much longer time scale than the initial phase separation the structures become coarser: either by merging of particles or by the growth of bigger particles at the cost of smaller ones. Numerical simulations of these phenomena are shown in Section 6. There is an extensive mathematical literature on the Cahn-Hilliard equation for reviews see [11,23].

In many systems the elastic behaviour of the two components making up the alloy is different. Hence, the two phases might have different elastic properties: as, for example, due to different lattice spacing. The resulting elastic effects have a pronounced impact on the evolving coarsening morphology and hence on the material properties. Figure 1 gives a first impression on how elastic

\footnotetext{
Email: harald,rumpf,wkd@iam.uni-bonn.de
} 
effects change the behaviour of the system. The complete sequences are shown in Section 6 . Although these effects have been studied a lot in the materials science and physics literature (see [15] for an overview) only very few mathematical results have appeared on the Cahn-Hilliard model with elastic effects (see, for example, $[6,17])$. The Cahn-Hilliard model with elastic contributions

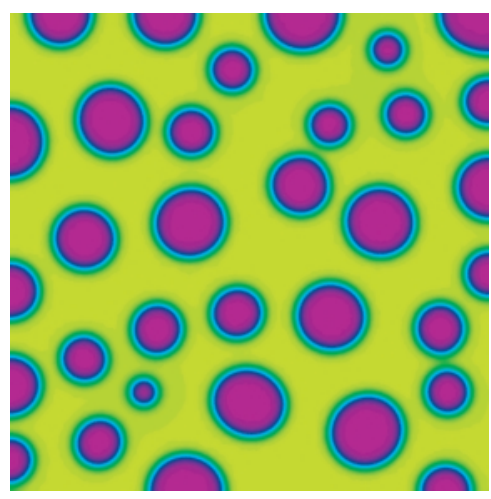

(a)

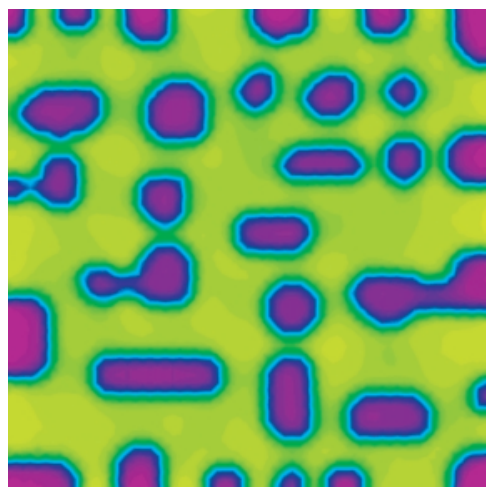

(b)

FIG. 1. Impact of elasticity on particle shape (numerical results). (a) Standard Cahn-Hilliard model, $t=0.5$ (b) CahnHilliard model with elasticity, $t=0.5$.

is based on a Ginzburg-Landau free energy which is a functional in terms of the concentration difference $\rho=\rho_{A}-\rho_{B}\left(\rho_{A}, \rho_{B} \in[0,1]\right.$ with $\rho_{A}+\rho_{B}=1$ being the concentrations of the two components) and the displacement field $\boldsymbol{u}$. Both functions shall be defined on a bounded domain $\Omega \subset \mathbb{R}^{d}$ with a sufficiently smooth boundary.

Now the Ginzburg-Landau free energy $E$ is defined to be

$$
E(\rho, \boldsymbol{u}):=\int_{\Omega}\left\{\psi(\rho)+\frac{\gamma}{2}|\nabla \rho|^{2}+W(\rho, \boldsymbol{u})\right\} \mathrm{d} x .
$$

The energy consists of three terms. The first term $\psi(\rho)$ is the chemical energy, which typically has a double well form taken in this paper to be

$$
\psi(\rho)=\frac{1}{4}\left(\rho^{2}-b^{2}\right)^{2}
$$

with a constant $b \in(0,1]$. We note that the system is locally in one of the two phases if the value of the concentration difference is close to one of the two minima $\pm b$ of $\psi$.

The second one describes the interfacial energy of the system and it is assumed that the parameter $\gamma$ is positive which implies that gradients are penalized. The effect of this term is that the total amount of transition zones is accounted for in the energy. This can be made precise in the limit when the thickness of the interfaces tends to zero (see [21]).

The third term accounts for energy contributions due to elastic effects. Since the deformations that appear in applications are usually small, the theory is based on linear elasticity and therefore the strain tensor is given by

$$
\mathcal{E}(\boldsymbol{u}):=\frac{1}{2}\left(\nabla \boldsymbol{u}+(\nabla \boldsymbol{u})^{t}\right)
$$


In the case of homogeneous elasticity, i.e. in the case that the elastic constants in the two phases are the same, the elastic energy is (see, for example, $[14,15,19]$ )

$$
W(\rho, \boldsymbol{u})=(\mathcal{E}(\boldsymbol{u})-\overline{\mathcal{E}}(\rho)): \mathcal{C}(\mathcal{E}(\boldsymbol{u})-\overline{\mathcal{E}}(\rho)) .
$$

Here, $\mathcal{C}$ is the possibly anisotropic elasticity tensor which we assume to be positive definite and complying with the usual symmetry conditions of linear elasticity. The term $\overline{\mathcal{E}}(\rho)$ is the stress-free strain at a concentration $\rho$. This is the value the strain tensor would take if the material is uniform with concentration $\rho$. We will assume that Vegard's law is satisfied, i.e. the stress-free strain is isotropic and varies linearly with the concentration. Hence (see [15]),

$$
\overline{\mathcal{E}}(\rho)=e(\rho-\bar{\rho}) \mathbf{1}
$$

with constants $e$ and $\bar{\rho}$. In the following we will take $\bar{\rho}=0$ which means that we take a reference state that is a uniform mixture of the two components. The product $A: B$ of two $d \times d$ matrices $A, B$ is defined to be the $\sum_{i, j=1}^{d} A_{i j} B_{i j}$.

Now the diffusion equation for the concentration $\rho$ is given by

$$
\frac{\partial \rho}{\partial t}=\Delta w \quad \text { in } \quad \Omega_{T}:=\Omega \times(0, T),
$$

where $T>0$ is an arbitrary but fixed time, while we have rescaled such that the mobility is equal to one. In the equation above we denote by $w$ the chemical potential difference which is given as the variational derivative $\frac{\delta E}{\delta \rho}$ of $E$ with respect to $\rho$. Since $\frac{\delta E}{\delta \rho}$ is defined by $\int_{\Omega} \frac{\delta E}{\delta \rho}(\rho, \boldsymbol{u}) \zeta=$ $\frac{\mathrm{d}}{\mathrm{d} \varepsilon} E(\rho+\varepsilon \zeta, \boldsymbol{u})_{\mid \varepsilon=0}$ for variations $\zeta$ we obtain

$$
w=-\gamma \Delta \rho+\psi^{\prime}(\rho)-\mathcal{S}: \overline{\mathcal{E}}^{\prime}(\rho) \quad \text { in } \quad \Omega_{T},
$$

where

$$
\mathcal{S}=\mathcal{C}(\mathcal{E}(\boldsymbol{u})-\overline{\mathcal{E}}(\rho))
$$

is the stress tensor. Since the relaxation into mechanical equilibrium occurs on a time scale that is fast compared to the time scale at which diffusion takes place we assume quasistatic equilibrium for the deformation. Hence, $\frac{\delta E}{\delta \boldsymbol{u}}=0$ which implies $\operatorname{div} \mathcal{S}=0$. Summing up the system comprises the following two equations for $\rho$ and $\boldsymbol{u}$ on $\Omega_{T}$ :

$$
\begin{aligned}
\partial_{t} \rho & =\Delta\left(\psi^{\prime}(\rho)-\gamma \Delta \rho-\mathcal{S}: \overline{\mathcal{E}}^{\prime}(\rho)\right), \\
0 & =\operatorname{div}(\mathcal{C}(\mathcal{E}(\boldsymbol{u})-\overline{\mathcal{E}}(\rho))) .
\end{aligned}
$$

The system is supplemented with the following boundary and initial conditions:

$$
\begin{aligned}
& \nabla \rho \cdot v=0, \quad \nabla w \cdot v=0, \quad \mathcal{S} v=0 \quad \text { on } \quad \partial \Omega \times(0, T), \\
& \rho(., 0)=\rho_{0}(.) \quad \text { in } \quad \Omega \text {, }
\end{aligned}
$$

where $v$ is the outer unit normal to $\partial \Omega$ and $\rho_{0} \in H^{1}(\Omega)$ are the initial data. For an existence and uniqueness result for the problem (1.3)-(1.7), see [17]. 
We remark that with the boundary conditions (1.6) mass is conserved and that the GinzburgLandau free energy is a Lyapunov functional, i.e. we have

$$
\frac{\mathrm{d}}{\mathrm{d} t} \int_{\Omega} \rho(x, t) \mathrm{d} x=0 \quad \text { and } \quad \frac{\mathrm{d}}{\mathrm{d} t} E(\rho(t), \boldsymbol{u}(t)) \leqslant 0 .
$$

In Fig. 2 we plot how the different parts of the energy evolve in time. The numerical simulation upon which the results are based used an initial value which was a random perturbation of a uniform mixture $\rho \equiv$ const. In the beginning the chemical energy decreases whereas the interfacial energy increases. This is due to the fact that during phase separation $\rho$ attains values which are at large portions of the domain close to the minima of the chemical energy $\psi$. As the transition zones between these regions are characterized by large gradients of $\rho$ the Dirichlet energy given by the second term in (1.2) increases.

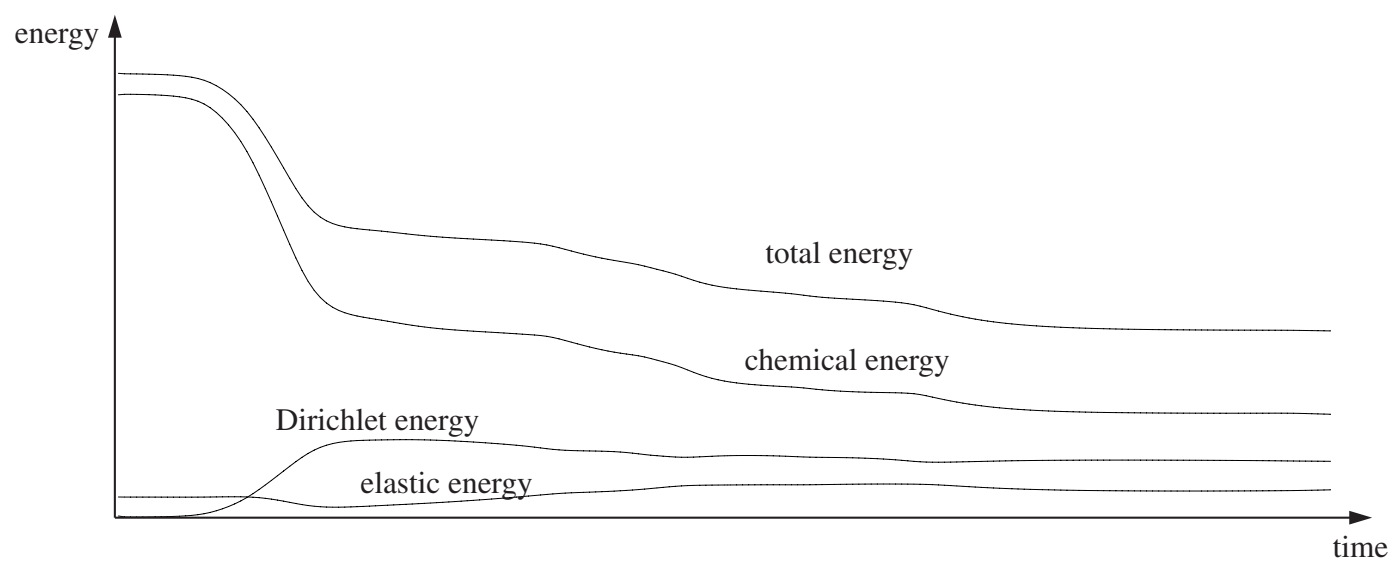

FIG. 2. Evolution of the different forms of energy (numerical results).

In the second stage of the evolution when the structures become coarser, the total amount of transition zones decreases. Correspondingly the amount of interfacial energy becomes smaller again. For the same reason the chemical energy decreases but at a much slower rate than during the initial phase.

One also observes that at later stages of the evolution the elastic energy part becomes larger compared to the two other ones. This is the case since for larger particles elastic energy contributions are bigger compared to the interfacial energy, whereas for small particles it is vice versa (see, for example, [15]).

At first, we will introduce a semi-discrete finite element approximation for the Cahn-Hilliard equation with elasticity. The discretization is based on an ansatz with continuous, piecewise linear finite elements for $\rho, w$ and $\boldsymbol{u}$ (see [12] for the case of the Cahn-Hilliard equation without elasticity). For the discretization in time we state two possibilities. One is the standard implicit Euler scheme and the second one is the strongly A-stable $\theta$-splitting scheme (see [3,22]). For the semi-discrete scheme we will show optimal error estimates in $L^{2}$ which generalizes results of Elliott et al. [12] to the case that elastic effects are included into the Cahn-Hilliard model. 
In the case of the implicit Euler scheme, we state a Lyapunov property of a discrete free energy and an error estimate for the fully discrete scheme (again compare [11] for the case without elasticity).

For practical computations we choose the $\theta$-splitting scheme which-due to its stability properties-allows for large time steps which are practically independent of the space discretization (see Table 1). The efficiency of our approach is further increased by an adaptive grid refinement and coarsening strategy. Here, we used a heuristic strategy which refines in interfacial regions and coarsens in the pure phases.

A major part of the paper is devoted to the presentation of numerical simulations showing several qualitative properties of solutions of the Cahn-Hilliard equation with elasticity. First we show a splitting phenomenon demonstrating that inverse coarsening can happen locally for some time already in the case of the Cahn-Hilliard equation without elasticity. We then demonstrate that a cubic anisotropy in the elastic energy has a pronounced effect on the particle shape. With no elastic contribution to the energy the phase boundaries tend to be round, whereas with elasticity a tendency towards a rectangular (or cubic) shape can be observed which is stronger for larger particles. Finally, we made computations setting the gradient energy coefficient $\gamma$ to zero. If also the elastic energy part is zero, the resulting evolution equation would be a second-order forwardbackward parabolic equation which consequently would be ill-posed. We now observe that in the numerical experiments with $\gamma=0$ and elastic effects present the elastic part has a regularizing effect. The numerical simulations show that rectangular phase regions appear which are separated by sharp interfaces. This regularizing effect is remarkable and deserves further study.

Finally, let us mention that there have been several numerical studies for the Cahn-Hilliard equation with elasticity which were based on Fourier transforms and spectral theory: see $[9,18,20]$ and the references therein for these approaches. For the numerical analysis of the Cahn-Hilliard equation with a more physical logarithmic free energy, see $[1,2,8]$.

\section{Weak formulation and discretization}

Testing the equations (1.3)-(1.5) with functions in $H^{1}(\Omega)$ yields the weak formulation of the problem:

Find

$$
\begin{gathered}
\rho \in L^{2}\left(0, T ; H^{1}(\Omega)\right) \cap H^{1}\left(0, T ;\left(H^{1}(\Omega)\right)^{*}\right), \\
w \in L^{2}\left(0, T ; H^{1}(\Omega)\right), \\
\boldsymbol{u} \in L^{2}\left(0, T ;\left(H^{1}(\Omega)\right)^{d}\right)
\end{gathered}
$$

such that for almost all $t \in(0, T)$ and for any $\zeta \in H^{1}(\Omega)$ and any $\xi \in\left(H^{1}\right)^{d}(\Omega)$

$$
\begin{aligned}
\left\langle\partial_{t} \rho, \zeta\right\rangle+(\nabla w, \nabla \zeta) & =0 \\
\gamma(\nabla \rho, \nabla \zeta)-\left(\mathcal{S}: \overline{\mathcal{E}}^{\prime}(\rho), \zeta\right) & =\left(w-\psi^{\prime}(\rho), \zeta\right), \\
((\mathcal{C}(\mathcal{E}-\overline{\mathcal{E}}(\rho))), \mathcal{E}(\boldsymbol{\xi})) & =0, \\
\rho(\cdot, 0) & =\rho_{0} \text { a.e. in } \Omega
\end{aligned}
$$

where (., .) is the $L^{2}$-scalar product and $\langle.,$.$\rangle is the duality pairing between the Sobolev space$ $H^{1}(\Omega)$ and its dual $H^{1}(\Omega)^{*}$. 
As the operator $\mathcal{E}$ has the nontrivial kernel

$$
\mathcal{K}:=\left\{s: \Omega \longrightarrow \mathbb{R}^{d} \mid s(x)=A x+b, \text { with } A \in \mathbb{R}^{d \times d} \text { skew symmetric and } b \in \mathbb{R}^{d}\right\}
$$

the displacement $\boldsymbol{u}$ is not uniquely determined. The source of this non-uniqueness is that translations and infinitesimal rotations have no impact on the elastic energy. We will always choose that solution of the elastic equation which has minimal $\left(L^{2}\right)^{d}(\Omega)$-norm. Since only $\mathcal{E}(\boldsymbol{u})$ enters the equation for $\rho$ there is still uniqueness in $\rho$ which is the quantity we are mainly interested in (see also [17]).

In the following we will assume for simplicity that $\Omega$ is a polyhedral domain. Generalizations to curved domains are of course possible by using boundary finite elements with curved faces (see, e.g., [7]). For a given triangulation $\mathcal{T}^{h}$ — which we suppose to be regular in the sense of [7]-we choose

$$
S^{h}:=\left\{\varphi \in C^{0}(\bar{\Omega})|\varphi|_{T} \in P_{1}(T) \forall T \in \mathcal{T}^{h}\right\} \subset H^{1}(\Omega)
$$

and $\left(S^{h}\right)^{d}$ as ansatz spaces for the finite element approximation of $\rho, w$, and $\boldsymbol{u}$ respectively. Here, we denote by $P_{1}(T)$ the set of all affine linear functions on $T$. To write the elastic terms more conveniently we introduce the following scalar product of two matrix-valued functions $\mathcal{A}$ and $\mathcal{B}$ :

$$
\langle\mathcal{A}, \mathcal{B}\rangle_{\mathcal{C}}:=(\mathcal{A}, \mathcal{C B})=\int_{\Omega} \mathcal{A}: \mathcal{C B}
$$

For later usage we introduce the corresponding norm $\|\cdot\|_{\mathcal{C}}:=\sqrt{\langle\cdot, \cdot\rangle_{\mathcal{C}}}$ and the corresponding scalar product with numerical integration $\langle\cdot, \cdot\rangle_{\mathcal{C}}^{h}$. The quadrature formula is assumed to be exact for piecewise linear integrands. Furthermore, we use the lumped mass scalar product $(., .)^{h}$ instead of the $L^{2}$-scalar product where appropriate.

Using these notations the standard finite element approximation of the elastic part of the problem leads to:

Given $\rho^{h}$ find $\boldsymbol{u}^{h}$ with $\boldsymbol{u}^{h}(\cdot, t) \in\left(S^{h}\right)^{d}$, such that

$$
\left\langle\mathcal{E}\left(\boldsymbol{u}^{h}\right), \mathcal{E}(\boldsymbol{\xi})\right\rangle_{\mathcal{C}}^{h}=\left\langle\overline{\mathcal{E}}\left(\rho^{h}\right), \mathcal{E}(\boldsymbol{\xi})\right\rangle_{\mathcal{C}}^{h} \quad \forall \boldsymbol{\xi} \in\left(S^{h}\right)^{d} .
$$

Note that all integrations in the identity above only involve piecewise linear integrands. Hence, the $\langle\cdot, \cdot\rangle_{\mathcal{C}}$-scalar product could be considered instead of the lumped mass scalar product $\langle\cdot, \cdot\rangle_{\mathcal{C}}^{h}$.

As $\overline{\mathcal{E}}(\rho)=e \rho \mathbf{1}$ we verify for any $\varphi \in H^{1}$ :

$$
\left(\mathcal{S}: \overline{\mathcal{E}}^{\prime}(\rho), \varphi\right)=\int_{\Omega}(\mathcal{S}: e \mathbf{1}) \varphi=\int_{\Omega} \mathcal{C}(\mathcal{E}(\boldsymbol{u})-\overline{\mathcal{E}}(\rho)): e \varphi \mathbf{1}=\langle\mathcal{E}(\boldsymbol{u})-\overline{\mathcal{E}}(\rho), \overline{\mathcal{E}}(\varphi)\rangle_{\mathcal{C}}
$$

Thus, using numerical quadrature the considered semi-discrete approximation scheme reads as follows:

(P) Find $\rho^{h}, w^{h}, \boldsymbol{u}^{h}$ with

such that

$$
\rho^{h}(\cdot, t), w^{h}(\cdot, t) \in S^{h}, \rho^{h}(x, \cdot) \in C^{1}([0, T]), \boldsymbol{u}^{h}(\cdot, t) \in\left(S^{h}\right)^{d},
$$

$$
\begin{aligned}
& 0=\left(\partial_{t} \rho^{h}, \varphi\right)^{h}+\left(\nabla w^{h}, \nabla \varphi\right), \\
& 0=\left(\psi^{\prime}\left(\rho^{h}\right)-w^{h}, \varphi\right)^{h}+\gamma\left(\nabla \rho^{h}, \nabla \varphi\right)-\left\langle\mathcal{E}\left(\boldsymbol{u}^{h}\right)-\overline{\mathcal{E}}\left(\rho^{h}\right), \overline{\mathcal{E}}(\varphi)\right\rangle_{\mathcal{C}}^{h} \\
& 0=\left\langle\mathcal{E}\left(\boldsymbol{u}^{h}\right)-\overline{\mathcal{E}}\left(\rho^{h}\right), \mathcal{E}(\boldsymbol{\xi})\right\rangle_{\mathcal{C}}^{h}
\end{aligned}
$$


holds for all $\varphi \in S^{h}$ and all $\boldsymbol{\xi} \in\left(S^{h}\right)^{d}$, and $\rho^{h}(x, 0)=\rho_{0}^{h}(x), \quad \forall x \in \Omega$.

Here, $\rho_{0}^{h}$ is assumed to be a suitable approximation of $\rho_{0}$ in $S^{h}$. For a fully discrete scheme we need to approximate the time derivative $\partial_{t} \rho^{h}$. This will be discussed in detail in Section 4 . Introducing the notation $\tilde{\mathcal{M}}$ for the mass matrix, $\mathcal{M}$ for the lumped mass matrix, $\mathcal{A}$ for the stiffness matrix and defining

$$
\mathcal{G}_{i j}:=\left\langle\mathcal{E}\left(\boldsymbol{\xi}_{i}\right), \mathcal{E}\left(\boldsymbol{\xi}_{j}\right)\right\rangle_{\mathcal{C}}^{h}=\int_{\Omega} \mathcal{E}\left(\boldsymbol{\xi}_{i}\right): \mathcal{C} \mathcal{E}\left(\boldsymbol{\xi}_{j}\right) \quad 1 \leqslant i, j \leqslant d \cdot N
$$

and

$$
\overline{\mathcal{G}}_{i j}:=\left\langle\mathcal{E}\left(\boldsymbol{\xi}_{i}\right), \overline{\mathcal{E}}\left(\varphi_{j}\right)\right\rangle_{\mathcal{C}}^{h}=\int_{\Omega} \mathcal{E}\left(\boldsymbol{\xi}_{i}\right): \mathcal{C} \overline{\mathcal{E}}\left(\varphi_{j}\right) \quad 1 \leqslant i \leqslant d \cdot N, 1 \leqslant j \leqslant N,
$$

where $\left\{\varphi_{i}\right\},\left\{\boldsymbol{\xi}_{i}\right\}$ are the standard basis functions of $S^{h}$ and $\left(S^{h}\right)^{d}$ respectively, while $N$ is the number of nodes of the triangulation $\mathcal{T}^{h}$. We formulate our semidiscrete scheme in matrix notation, splitting the right-hand side into a nonlinear and a linear term:

$$
\mathcal{M} \partial_{t} \vec{\rho}^{h}=-\mathcal{A} \overrightarrow{\psi^{\prime}\left(\rho^{h}\right)}-\mathcal{L} \vec{\rho}^{h}
$$

where

$$
\mathcal{L}:=\left(\gamma \mathcal{A} \mathcal{M}^{-1} \mathcal{A}+e^{2}(\mathbf{1}: \mathcal{C} \mathbf{1}) \mathcal{A}-\mathcal{A M}^{-1} \overline{\mathcal{G}}^{T} \mathcal{G}^{-1} \overline{\mathcal{G}}\right) .
$$

Here an overhead arrow distinguishes a vector of nodal values from the corresponding finite element function. Since the matrix $\mathcal{G}$ does not have full rank, the inverse does not exist in the usual sense. Hence, we denote the solution operator of the elastic system — which selects the solution with minimal $\left(L^{2}\right)^{d}(\Omega)$-norm—by $\mathcal{G}^{-1}$.

\section{Convergence of the semidiscrete scheme}

Let us now consider the convergence properties of semidiscrete solutions of $\left(\mathbf{P}^{\mathbf{h}}\right)$. We obtain the following theorem that generalizes the convergence results obtained by Elliott et al. [12] for the standard Cahn-Hilliard model.

THEOREM 1 (A priori ERROR ESTIMATE FOR THE SEMI-DISCRETE SCHEME.) Let $\rho, w$ and $\boldsymbol{u}$ be solutions of the Cahn-Hilliard equation with elasticity on a convex polyhedral bounded domain $\Omega \subset \mathbb{R}^{d}(d \in\{1,2,3\})$ with regularity

$$
\begin{aligned}
\rho & \in L^{\infty}\left(0, T ; H^{2}(\Omega)\right) \\
\partial_{t} \rho & \in L^{\infty}\left(0, T ; H^{1}(\Omega)\right) \\
\boldsymbol{u} & \in L^{\infty}\left(0, T ;\left(H^{2}(\Omega)\right)^{d}\right)
\end{aligned}
$$

and suppose $\left\|\rho_{0}-\rho_{0}^{h}\right\|_{L^{2}(\Omega)} \leqslant c h^{2}$.

Then the following error bounds hold:

$$
\begin{aligned}
& \left\|\rho-\rho^{h}\right\|_{L^{\infty}\left(L^{2}(\Omega)\right)}+\left\|w-w^{h}\right\|_{L^{2}\left(L^{2}(\Omega)\right)}+\left\|\partial_{t} \rho-\partial_{t} \rho^{h}\right\|_{L^{2}\left(L^{2}(\Omega)\right)} \leqslant c h^{2}, \\
& \left\|\rho-\rho^{h}\right\|_{L^{\infty}\left(H^{1,2}(\Omega)\right)}+\left\|w-w^{h}\right\|_{L^{2}\left(H^{1,2}(\Omega)\right)} \leqslant c h, \\
& \left\|\mathcal{E}\left(\boldsymbol{u}-\boldsymbol{u}^{h}\right)\right\|_{L^{\infty}\left(0, T ;\left(L^{2}\right)^{d}\right)} \leqslant c h .
\end{aligned}
$$


Proof. Let $\bar{w}^{h} \in S^{h}$ be the generalized Ritz projection of $w$, i.e. $\left(\bar{w}^{h}-w, 1\right)=0$ and for any $\varphi \in S^{h}$

$$
\left(\nabla \bar{w}^{h}, \nabla \varphi\right)=\left(-\Delta w+\delta_{1}^{h}, \varphi\right)^{h} .
$$

Here, $\delta_{1}^{h} \in \mathbb{R}$ is defined as $\frac{(\Delta w, 1)^{h}}{(1,1)^{h}}$ in order to guarantee existence of $\bar{w}^{h}$. Since $\int_{\Omega} \Delta w=0$ the properties of the lumped mass scalar product imply

$$
\left|\delta_{1}^{h}\right| \leqslant c h^{2}\|\Delta w\|_{H^{1}} \leqslant c h^{2} .
$$

The last inequality follows because $\nabla \Delta w=\nabla \partial_{t} \rho \in L^{\infty}\left(0, T ; L^{2}(\Omega)\right)$. We then split the error $w-w^{h}$ into two parts to be treated separately:

$$
\theta^{w}:=w^{h}-\bar{w}^{h} \quad \kappa^{w}:=\bar{w}^{h}-w \quad \text { i.e. } \quad w^{h}-w=\theta^{w}+\kappa^{w} .
$$

Standard properties of the Ritz projection imply an estimate for $\kappa^{w}$

$$
\left\|\kappa^{w}\right\|_{L^{2}(\Omega)}+h\left\|\nabla \kappa^{w}\right\|_{L^{2}(\Omega)} \leqslant c h^{2} .
$$

The error part $\theta^{w}$ will be estimated later. We introduce the following two bilinear forms on $\left(H^{1}(\Omega)\right)^{d+1} \times\left(H^{1}(\Omega)\right)^{d+1}$ :

$$
\begin{aligned}
\mathcal{B}((\varphi, \boldsymbol{\xi}),(\zeta, \boldsymbol{\sigma})) & :=\gamma(\nabla \varphi, \nabla \zeta)+\langle\mathcal{E}(\boldsymbol{\xi})-\overline{\mathcal{E}}(\varphi), \mathcal{E}(\boldsymbol{\sigma})-\overline{\mathcal{E}}(\zeta)\rangle_{\mathcal{C}}, \\
\mathcal{B}_{h}((\varphi, \boldsymbol{\xi}),(\zeta, \boldsymbol{\sigma})) & :=\gamma(\nabla \varphi, \nabla \zeta)+\langle\mathcal{E}(\boldsymbol{\xi})-\overline{\mathcal{E}}(\varphi), \mathcal{E}(\boldsymbol{\sigma})-\overline{\mathcal{E}}(\zeta)\rangle_{\mathcal{C}}^{h},
\end{aligned}
$$

where $\varphi, \zeta \in H^{1}(\Omega)$ and $\boldsymbol{\xi}, \boldsymbol{\sigma} \in\left(H^{1}\right)^{d}(\Omega)$. Connected with the bilinear form $\mathcal{B}$ is a semi-norm $\|(\rho, \boldsymbol{u})\|_{\mathcal{B}}:=\sqrt{\mathcal{B}((\rho, \boldsymbol{u}),(\rho, \boldsymbol{u}))}$ on $\left(H^{1}\right)^{d+1}(\Omega)$ and for $\mathcal{B}_{h}$ a respective semi-norm $\|\cdot\|_{\mathcal{B}_{h}}$. Using the new notation we can write the elliptic problem (2.2), (2.3) as

$$
\mathcal{B}((\rho, \boldsymbol{u}),(\zeta, \boldsymbol{\sigma}))=\left(w-\psi^{\prime}(\rho), \zeta\right) \quad \forall(\zeta, \boldsymbol{\sigma}) \in\left(H^{1}(\Omega)\right)^{d+1}
$$

Now we consider the following two auxiliary problems:

$\left(\hat{\mathbf{P}}^{\mathbf{h}}\right) \quad$ Find $\hat{\rho}^{h} \in S^{h}$ and $\hat{\boldsymbol{u}}^{h} \in\left(S^{h}\right)^{d}$ such that for any $(\varphi, \boldsymbol{\xi}) \in\left(S^{h}\right)^{d+1}$

$$
\mathcal{B}\left(\left(\hat{\rho}^{h}, \hat{\boldsymbol{u}}^{h}\right),(\varphi, \boldsymbol{\xi})\right)=\left(w-\psi^{\prime}(\rho), \varphi\right)
$$

and
$\left(\overline{\mathbf{P}}^{\mathbf{h}}\right) \quad$ Find $\bar{\rho}^{h} \in S^{h}$ and $\overline{\boldsymbol{u}}^{h} \in\left(S^{h}\right)^{d}$ such that for any $(\varphi, \boldsymbol{\xi}) \in\left(S^{h}\right)^{d+1}$

$$
\mathcal{B}_{h}\left(\left(\bar{\rho}^{h}, \overline{\boldsymbol{u}}^{h}\right),(\varphi, \boldsymbol{\xi})\right)=\left(\bar{w}^{h}-\psi^{\prime}(\rho)+\delta_{2}^{h}, \varphi\right)^{h}
$$

with $\delta_{2}^{h}:=\frac{\left(\psi^{\prime}(\rho)-\bar{w}^{h}, 1\right)^{h}}{(1,1)^{h}}$. We remark that problem $\left(\hat{\mathbf{P}}^{\mathbf{h}}\right)$ is solvable since the solvability condition $\left(w-\psi^{\prime}(\rho), 1\right)=0$ is fulfilled. This can be seen by choosing $\zeta \equiv 1$ and $\sigma=e \mathbf{1}$ in (3.3). Whereas problem $\left(\overline{\mathbf{P}}^{\mathbf{h}}\right)$ is solvable due to the definition of $\delta_{2}^{h}$, which can be seen by taking $\varphi \equiv 1$ and $\boldsymbol{\xi}=e \mathbf{1}$ in (3.5). Solutions of both problems are not unique and therefore we can choose $\bar{\rho}^{h}, \hat{\rho}^{h}$ such that $\int_{\Omega} \bar{\rho}^{h}=\int_{\Omega} \hat{\rho}^{h}=\int_{\Omega} \rho$ and $\overline{\boldsymbol{u}}^{h}, \hat{\boldsymbol{u}}^{h}$ are assumed to be the solutions with minimal $\left(L^{2}\right)^{d}$-norm.

We split the error $\rho^{h}-\rho$ and $\boldsymbol{u}^{h}-\boldsymbol{u}$ in three parts:

$$
\begin{array}{lll}
\theta^{\rho}:=\rho^{h}-\bar{\rho}^{h} & \kappa^{\rho}:=\bar{\rho}^{h}-\hat{\rho}^{h} & \iota^{\rho}:=\hat{\rho}^{h}-\rho, \\
\boldsymbol{\theta}^{\boldsymbol{u}}:=\boldsymbol{u}^{h}-\overline{\boldsymbol{u}}^{h} & \boldsymbol{\kappa}^{\boldsymbol{u}}:=\overline{\boldsymbol{u}}^{h}-\hat{\boldsymbol{u}}^{h} & \boldsymbol{\iota}^{\boldsymbol{u}}:=\hat{\boldsymbol{u}}^{h}-\boldsymbol{u} .
\end{array}
$$


In order to estimate $\kappa^{\rho}$ and $\kappa^{u}$ we subtract (3.4) from (3.5) and get

$$
\begin{aligned}
\left(\bar{w}^{h}-\psi^{\prime}(\rho)+\delta_{2}^{h}, \varphi\right)^{h}- & \left(w-\psi^{\prime}(\rho), \varphi\right)=\mathcal{B}_{h}\left(\left(\bar{\rho}^{h}, \overline{\boldsymbol{u}}^{h}\right),(\varphi, \boldsymbol{\xi})\right)-\mathcal{B}\left(\left(\hat{\rho}^{h}, \hat{\boldsymbol{u}}^{h}\right),(\varphi, \boldsymbol{\xi})\right) \\
= & \gamma\left(\nabla \kappa^{\rho}, \nabla \varphi\right)+\left\langle\mathcal{E}\left(\boldsymbol{\kappa}^{\boldsymbol{u}}\right)-\overline{\mathcal{E}}\left(\kappa^{\rho}\right), \mathcal{E}(\boldsymbol{\xi})-\overline{\mathcal{E}}(\varphi)\right\rangle_{\mathcal{C}}^{h} \\
& +e^{2}(\mathbf{1}: \mathcal{C} \mathbf{1})\left[\left(\hat{\rho}^{h}, \varphi\right)^{h}-\left(\hat{\rho}^{h}, \varphi\right)\right]
\end{aligned}
$$

for any $(\varphi, \boldsymbol{\xi}) \in\left(S^{h}\right)^{d+1}$, since the quadrature formula of the $\langle\cdot, \cdot\rangle_{\mathcal{C}}^{h}$-scalar product is assumed to be exact for piecewise constant and piecewise linear functions. Choosing $\varphi=\kappa^{\rho}$ and $\boldsymbol{\xi}=\boldsymbol{\kappa}^{\boldsymbol{u}}$ yields with the properties of the lumped mass scalar product [25]:

$$
\begin{aligned}
\gamma & \left\|\nabla \kappa^{\rho}\right\|_{L^{2}(\Omega)}^{2}+\left(\left\|\mathcal{E}\left(\kappa^{\boldsymbol{u}}\right)-\overline{\mathcal{E}}\left(\kappa^{\rho}\right)\right\|_{\mathcal{C}}^{h}\right)^{2} \\
& =\left(\bar{w}^{h}-\psi^{\prime}(\rho)+\delta_{2}^{h}, \kappa^{\rho}\right)^{h}-\left(w-\psi^{\prime}(\rho), \kappa^{\rho}\right)+e^{2}(\mathbf{1}: \mathcal{C} \mathbf{1})\left[\left(\hat{\rho}^{h}, \kappa^{\rho}\right)-\left(\hat{\rho}^{h}, \kappa^{\rho}\right)^{h}\right] \\
& \leqslant\left[c h^{2}\|w\|_{H^{2,2}(\Omega)}+\left|\kappa^{w}\right|_{h}+c h^{2}\left\|\psi^{\prime}(\rho)\right\|_{H^{2,2}(\Omega)}+\left|\delta_{2}^{h}\right|_{h}+c h^{2}\left\|\hat{\rho}^{h}\right\|_{H^{1,2}(\Omega)}\right]\left\|\kappa^{\rho}\right\|_{H^{1,2}(\Omega)} .
\end{aligned}
$$

From the definition of $\delta_{2}^{h}$ it follows that

$$
\left|\delta_{2}^{h}\right| \leqslant c h^{2}\left(\left\|\psi^{\prime}(\rho)\right\|_{H^{1,2}(\Omega)}+\left\|\kappa^{w}\right\|_{H^{1,2}(\Omega)}+\|w\|_{H^{1,2}(\Omega)}\right) \leqslant c h^{2} .
$$

To show that $\hat{\rho}^{h}$ is bounded in the $H^{1}(\Omega)$-norm we test (3.4) with $\varphi=\hat{\rho}^{h}$ and $\boldsymbol{\xi}=\hat{\boldsymbol{u}}^{h}$ :

$$
\begin{aligned}
\gamma\left\|\nabla \hat{\rho}^{h}\right\|_{L^{2}(\Omega)}^{2} & +\left(\left\|\mathcal{E}\left(\hat{\boldsymbol{u}}^{h}\right)-\overline{\mathcal{E}}\left(\hat{\rho}^{h}\right)\right\|_{\mathcal{C}}^{h}\right)^{2}=\left(w-\psi^{\prime}(\rho), \hat{\rho}^{h}\right) \\
& \leqslant\left\|w-\psi^{\prime}(\rho)\right\|_{L^{2}(\Omega)}\left\|\hat{\rho}^{h}\right\|_{L^{2}(\Omega)} \\
& \leqslant\left\|w-\psi^{\prime}(\rho)\right\|_{L^{2}(\Omega)} c\left(\left\|\nabla \hat{\rho}^{h}\right\|_{L^{2}(\Omega)}+1\right) \\
& \leqslant \frac{1}{\varepsilon}\left\|w-\psi^{\prime}(\rho)\right\|_{L^{2}(\Omega)}^{2}+\varepsilon c\left\|\nabla \hat{\rho}^{h}\right\|_{L^{2}(\Omega)}^{2}+c\left\|w-\psi^{\prime}(\rho)\right\|_{L^{2}(\Omega)} .
\end{aligned}
$$

For appropriate $\varepsilon$ this implies that $\left\|\nabla \hat{\rho}^{h}\right\|_{L^{2}(\Omega)} \leqslant c$ independent of $h$. Thus, due to the Poincare inequality we obtain an estimate of $\kappa^{\rho}$ from (3.6):

$$
\left\|\nabla \kappa^{\rho}\right\|_{L^{2}(\Omega)} \leqslant c h^{2} \quad \text { and } \quad\left\|\kappa^{\rho}\right\|_{L^{2}(\Omega)} \leqslant c h^{2} .
$$

Concerning $\kappa^{u}$ we note that from (3.6) it follows that

$$
\begin{aligned}
\left\|\mathcal{E}\left(\kappa^{u}\right)\right\|_{L^{2}(\Omega)}^{2} & \leqslant c\left(\left\|\mathcal{E}\left(\kappa^{u}\right)\right\|_{\mathcal{C}}^{h}\right)^{2} \\
& \leqslant c\left(\left\|\overline{\mathcal{E}}\left(\kappa^{\rho}\right)\right\|_{\mathcal{C}}^{h}\right)^{2}+c\left(\left\|\mathcal{E}\left(\kappa^{u}\right)-\overline{\mathcal{E}}\left(\kappa^{\rho}\right)\right\|_{\mathcal{C}}^{h}\right)^{2} \\
& \leqslant c\left(\gamma\left\|\nabla \kappa^{\rho}\right\|_{L^{2}(\Omega)}^{2}+\left(\left\|\mathcal{E}\left(\kappa^{u}\right)-\overline{\mathcal{E}}\left(\kappa^{\rho}\right)\right\|_{\mathcal{C}}^{h}\right)^{2}\right) \\
& \leqslant c h^{4} .
\end{aligned}
$$


Thereby, $\left\|\mathcal{E}\left(\kappa^{u}\right)\right\|_{L^{2}(\Omega)} \leqslant c h^{2}$.

Now we turn to the Galerkin projection errors $\iota^{\rho}=\hat{\rho}^{h}-\rho$ and $\iota^{\boldsymbol{u}}=\hat{\boldsymbol{u}}^{h}-\boldsymbol{u}$. Combining equation (3.3) and (3.4) yields the usual Galerkin orthogonality relation

$$
\mathcal{B}\left(\left(\iota^{\rho}, \iota^{\boldsymbol{u}}\right),(\varphi, \boldsymbol{\xi})\right)=0
$$

for any $\varphi \in S^{h}$ and any $\boldsymbol{\xi} \in\left(S^{h}\right)^{d}$. Hence,

$$
\left\|\left(\iota^{\rho}, \iota^{\boldsymbol{u}}\right)\right\|_{\mathcal{B}} \leqslant \inf _{\varphi \in S^{h}, \boldsymbol{\xi} \in\left(S^{h}\right)^{d}}\|(\rho-\varphi, \boldsymbol{u}-\boldsymbol{\xi})\|_{\mathcal{B}},
$$

where the right-hand side is bounded by $c h$, and we achieve

$$
\left\|\nabla \iota^{\rho}\right\|_{L^{2}(\Omega)}+\left\|\mathcal{E}\left(\iota^{\boldsymbol{u}}\right)\right\|_{L^{2}(\Omega)} \leqslant c\left\|\left(\iota^{\rho}, \iota^{\boldsymbol{u}}\right)\right\|_{\mathcal{B}} \leqslant c h .
$$

To estimate the $L^{2}$-norm we make use of a standard duality argument [25]. Let $(\varphi, \boldsymbol{\xi}) \in$ $\left(L^{2}(\Omega)\right)^{d+1}$ which fulfil the solvability conditions

$$
\int_{\Omega} \boldsymbol{\xi} \cdot \boldsymbol{\sigma} \mathrm{d} x=0, \quad \int_{\Omega} \varphi \mathrm{d} x+e \int_{\Omega} \boldsymbol{\xi} \cdot x \mathrm{~d} x=0
$$

for all elements $\sigma$ that lie in the kernel $\mathcal{K}$ of the operator $\mathcal{E}$. Then we define $(\mu, \nu) \in\left(H^{1}(\Omega)\right)^{d+1}$ as the solution of the dual problem

$$
\begin{aligned}
-\gamma \Delta \mu-e \operatorname{tr} \mathcal{C}(\mathcal{E}(\boldsymbol{\nu})-\overline{\mathcal{E}}(\mu)) & =\varphi \\
-\operatorname{div} \mathcal{C}(\mathcal{E}(\boldsymbol{\nu})-\overline{\mathcal{E}}(\mu)) & =\boldsymbol{\xi}
\end{aligned}
$$

with Neumann boundary condition for $\mu$ and the stress-free boundary condition $\mathcal{C}(\mathcal{E}(\boldsymbol{\nu})-\overline{\mathcal{E}}(\mu)) v=0$. We compute

$$
\begin{aligned}
\left(\iota^{\rho}, \varphi\right)+\left(\iota^{\boldsymbol{u}}, \boldsymbol{\xi}\right) & =\mathcal{B}\left(\left(\iota^{\rho}, \boldsymbol{\iota}^{\boldsymbol{u}}\right),(\mu, \boldsymbol{\nu})\right) \\
& =\inf _{\mu^{h} \in S^{h}, \boldsymbol{\nu}^{h} \in\left(S^{h}\right)^{d}} \mathcal{B}\left(\left(\iota^{\rho}, \boldsymbol{\iota}^{\boldsymbol{u}}\right),\left(\mu-\mu^{h}, \boldsymbol{\nu}-\boldsymbol{\nu}^{h}\right)\right) \\
& \leqslant\left\|\left(\iota^{\rho}, \boldsymbol{\iota}^{\boldsymbol{u}}\right)\right\|_{\mathcal{B}} \inf _{\mu^{h} \in S^{h}, \boldsymbol{\nu}^{h} \in\left(S^{h}\right)^{d}}\left\|\left(\mu-\mu^{h}, \boldsymbol{\nu}-\boldsymbol{\nu}^{h}\right)\right\|_{\mathcal{B}} \\
& \leqslant c h^{2}\|(\mu, \boldsymbol{\nu})\|_{H^{2,2}(\Omega)} .
\end{aligned}
$$

Due to Korn's inequality and elliptic regularity theory we have $\|(\mu, \nu)\|_{H^{2,2}(\Omega)} \leqslant c\|(\varphi, \boldsymbol{\xi})\|_{L^{2}(\Omega)}$. Now we want to choose

$$
(\varphi, \boldsymbol{\xi})=\left(\iota^{\rho}-c^{h}, \iota^{\boldsymbol{u}}\right)=\left(\hat{\rho}^{h}-\rho-c^{h}, \hat{\boldsymbol{u}}^{h}-\boldsymbol{u}\right)
$$

$\left(c^{h}\right.$ a constant to be determined later) as the right-hand side in (3.8), (3.9) and therefore we have to check the solvability conditions (3.7). The displacements $\boldsymbol{u}$ and $\hat{\boldsymbol{u}}^{h}$ were chosen to have minimal $L^{2}$-norm under all solutions that lie in $\boldsymbol{u}+\mathcal{K}$ and $\hat{\boldsymbol{u}}^{h}+\mathcal{K}$ respectively. This implies that $\boldsymbol{u}$ and $\hat{\boldsymbol{u}}^{h}$ are orthogonal to $\mathcal{K}$ which shows that the first solvability condition in (3.7) holds. Choosing $c^{h}$ such that the second solvability condition in (3.7) is fulfiled for $(\varphi, \boldsymbol{\xi})$ and taking into account that $\rho$ and $\hat{\rho}^{h}$ have the same mean value gives

$$
\begin{aligned}
\left\|\iota^{\rho}\right\|_{L^{2}(\Omega)}^{2}+\left\|\iota^{\boldsymbol{u}}\right\|_{L^{2}(\Omega)}^{2} & =\left(\iota^{\rho}, \iota^{\rho}-c^{h}\right)+\left(\iota^{\boldsymbol{u}}, \boldsymbol{\iota}^{\boldsymbol{u}}\right) \\
& \leqslant c h^{2}\left\|\left(\iota^{\rho}-c^{h}, \iota^{\boldsymbol{u}}\right)\right\|_{L^{2}(\Omega)} .
\end{aligned}
$$


Since $c^{h}$ is bounded by $\left\|\left(\rho-\hat{\rho}^{h}, \boldsymbol{u}-\hat{\boldsymbol{u}}^{h}\right)\right\|_{L^{2}(\Omega)}$ we can conclude

$$
\begin{aligned}
\left\|\iota^{\rho}\right\|_{L^{2}(\Omega)}+h\left\|\nabla \iota^{\rho}\right\|_{L^{2}(\Omega)} & \leqslant c h^{2}, \\
\left\|\iota^{\boldsymbol{u}}\right\|_{L^{2}(\Omega)}+h\left\|\mathcal{E}\left(\iota^{\boldsymbol{u}}\right)\right\|_{L^{2}(\Omega)} & \leqslant c h^{2} .
\end{aligned}
$$

So far, the generalized projection errors $\iota^{\rho}, \iota^{u}$ and the error terms due to numerical quadrature $\kappa^{\rho}, \kappa^{u}$ are estimated in agreement with the stated result on the global error.

Concerning the estimates for the remaining error quantities $\theta^{\rho}, \theta^{w}$ and $\boldsymbol{\theta}^{\boldsymbol{u}}$ we can proceed as in the corresponding proof in [12] for the standard Cahn-Hilliard problem. Here, we have used that $\rho \in L^{\infty}\left(0, T ; H^{2}(\Omega)\right)$ and that $d \leqslant 3$ to apply the Sobolev embedding theorem to conclude that $\left\|\rho^{h}(\cdot, t)\right\|_{L^{\infty}(\Omega)}$ is uniformly bounded with respect to $t$. Therefore we obtain, as in [12], the following inequalities:

$$
\begin{aligned}
\left|\theta^{\rho}(t)\right|_{h}^{2}+\int_{0}^{t}\left|\theta^{w}(s)\right|_{h}^{2} \mathrm{~d} s & \leqslant C\left(h^{4}+\left|\theta^{\rho}(0)\right|_{h}^{2}\right) \\
\left\|\nabla \theta^{\rho}(t)\right\|_{L^{2}(\Omega)}^{2}+\left|\theta^{w}(t)\right|_{h}^{2} & +\int_{0}^{t}\left|\partial_{t} \theta^{\rho}(s)\right|_{h}^{2}+\left\|\nabla \theta^{w}(s)\right\|_{L^{2}(\Omega)}^{2} \mathrm{~d} s \\
& \leqslant C\left(h^{4}+\left\|\theta^{\rho}(0)\right\|_{H^{1,2}(\Omega)}^{2}+\left|\theta^{w}(0)\right|_{h}^{2}\right) .
\end{aligned}
$$

which hold for $t \in[0, T]$. From this we finally get estimates for $\Theta^{\rho}, \Theta^{w}$ and $\boldsymbol{\theta}^{\boldsymbol{u}}$ and, together with the already shown bounds for $\iota^{\rho}, \iota^{u}, \kappa^{\rho}, \kappa^{w}$ and $\kappa^{u}$, the theorem follows immediately.

Let us remark that in convex domains $H^{2}$-regularity results in space hold true, even if the boundary is not smooth. This implies that the assumptions in the theorem hold true if the initial data are sufficiently smooth. Furthermore, we point out that the error estimates are optimal in the sense that with the finite element method an order of convergence better than $\mathrm{O}\left(h^{2}\right)$ in the $L^{2}$-norm and $\mathrm{O}(h)$ in the $H^{1}$-norm cannot be expected anyway. We also note that standard arguments yield corresponding estimates in the $L^{\infty}$-norm. Furthermore, a generalization of these results to more general smooth energies $\psi$ and stress-free strains $\overline{\mathcal{E}}$ is possible.

\section{Discretization in time}

In Section 2 we introduced a spatial discretization of the Cahn-Hilliard model with elasticity and obtained a system of nonlinear ordinary differential equation (2.6). We will now focus onto an appropriate discretization in time. Replacing the time derivative $\partial_{t} \rho^{h}$ by a backward difference quotient $\frac{\rho^{h}(\cdot, t)-\rho^{h}(\cdot, t-\tau)}{\tau}$ with timestep $\tau$ we obtain an implicit Euler scheme. In each step we have to compute a new density vector $\vec{\rho}_{n+1}^{h}$ at time $(n+1) \tau$ by solving

$$
(\mathcal{M}+\tau \mathcal{L}) \vec{\rho}_{n+1}^{h}+\tau \mathcal{A} \overrightarrow{\psi^{\prime}\left(\rho_{n+1}^{h}\right)}=\mathcal{M} \vec{\rho}_{n}^{h} .
$$

This scheme is known to be first-order consistent (cf. the error estimate below (4.3)). We can improve the consistency order selecting a Crank-Nicolson type discretization. The nonlinear part is approximated by (see also $[10,16])$

$$
\phi(r, s):= \begin{cases}\frac{\psi(r)-\psi(s)}{r-s}, & \text { for } r \neq s \\ \psi^{\prime}(r), & \text { for } r=s\end{cases}
$$


and we have to solve in each timestep the nonlinear system

$$
\left(\mathcal{M}+\frac{\tau}{2} \mathcal{L}\right) \vec{\rho}_{n+1}^{h}+\frac{\tau}{2} \mathcal{A} \overrightarrow{\phi\left(\rho_{n+1}^{h}, \rho_{n}^{h}\right)}=\left(\mathcal{M}-\frac{\tau}{2} \mathcal{L}\right) \vec{\rho}_{n}^{h} .
$$

With respect to a proper numerical modelling it is important to guarantee properties known for the continuous solution also for its discrete counterpart. We immediately observe that mass is conserved by both discrete schemes. In the continuous case, the energy turns out to be a Lyapunov functional. The same holds true for the implicit Euler scheme. We obtain that under the assumptions $\psi^{\prime \prime}>-c_{0}$ and $\tau \leqslant \frac{8 \gamma}{c_{0}^{2}}$ the functional

$$
\hat{W}^{h}(\rho, \boldsymbol{u}):=\frac{\gamma}{2}|\rho|_{1}^{2}+(\psi(\rho), 1)^{h}+\frac{1}{2}\left(\|\mathcal{E}(\boldsymbol{u})-\overline{\mathcal{E}}(\rho)\|_{\mathcal{C}}^{h}\right)^{2}
$$

decays in time (cf. Fig. 2).

For the proof, which is a generalization of Elliott's proof [11] of the existence of a Lyapunov functional, see [27]. There an analogous result is proven also for a modified Crank-Nicolson scheme.

The convergence results presented in Section 3 for the semi-discrete scheme can easily be extended to the implicit Euler and the Crank-Nicolson scheme (see [11,13] for the standard CahnHilliard model). For details of the proof see [27]. We obtain an error estimate

$$
\left\|\rho_{n}^{h}-\rho(n \tau)\right\|_{L^{2}(\Omega)} \leqslant c\left(\left\|\rho_{0}-\rho_{0}^{h}\right\|_{L^{2}(\Omega)}+h^{2}+\tau^{s}\right),
$$

where $c$ is independent of $h$ and $\tau$. Depending on the time discretization, $s=1$ for the implicit Euler and $s=2$ for the Crank-Nicolson discretization.

Thus, the backward Euler discretization is only of first order, whereas the Crank-Nicolson scheme delivers a second-order discretization in time (cf. estimate (4.3)). Unfortunately the CrankNicolson time stepping scheme is not strongly A-stable [25]. Hence, high-frequency components in the function governed by the evolution equation are not smoothed. Phase separation as it occurs in the Cahn-Hilliard model is characterized by significant high-frequency contributions, visible at the interfaces. In fact, for larger timesteps, still much smaller than the spatial grid size, oscillations already show up in the implementation of the Crank-Nicolson scheme. Hence, we ask for a second order-time discretization which is applicable for our nonlinear evolution problem and which has the strong A-stability property.

The $\Theta$-splitting scheme, originally proposed by Strang [24] and as an operator splitting scheme by Bristeau et al. [3] for the Navier-Stokes equations has this property for a certain choice of control parameters.

In each timestep we have to successively solve the following three equation in $\vec{\rho}_{n+\theta}^{h}, \vec{\rho}_{n+1-\theta}^{h}$, and $\vec{\rho}_{n}^{h}$, for given $\theta \in(0,0.5)$ :

$$
\begin{aligned}
(\mathcal{M}+\alpha \theta \tau \mathcal{L}) \vec{\rho}_{n+\theta}^{h} & =(\mathcal{M}-\beta \theta \tau \mathcal{L}) \vec{\rho}_{n}^{h}-\theta \tau \mathcal{A} \overrightarrow{\psi^{\prime}\left(\vec{\rho}_{n}^{h}\right)}, \\
(\mathcal{M}+\beta(1-2 \theta) \tau \mathcal{L}) \vec{\rho}_{n+1-\theta}^{h}+(1-2 \theta) \tau \mathcal{A} \overrightarrow{\psi^{\prime}\left(\vec{\rho}_{n+1-\theta}^{h}\right)}=(\mathcal{M}-\alpha(1-2 \theta) \tau \mathcal{L}) \vec{\rho}_{n+\theta}^{h}, & \\
(\mathcal{M}+\alpha \theta \tau \mathcal{L}) \vec{\rho}_{n+1}^{h} & =(\mathcal{M}-\beta \theta \tau \mathcal{L}) \vec{\rho}_{n+1-\theta}^{h}-\theta \tau \mathcal{A} \overrightarrow{\psi^{\prime}\left(\vec{\rho}_{n+1-\theta}^{h}\right)}
\end{aligned}
$$


for a second parameter $\alpha \in(0.5,1]$ to be fixed and for $\beta=1-\alpha$.

Choosing $\theta=1-\frac{\sqrt{2}}{2}$ this scheme is of second-order accuracy. For a detailed analysis see [22]. Here, in the context of Cahn-Hilliard models we confine ourselves to numerical observations.

For the parameter $\theta=1-\frac{\sqrt{2}}{2}$ and $\alpha=\frac{1-2 \theta}{1-\theta} \approx 0.586$ we performed several experiments to evaluate a 'maximal' possible timestep for different initial values, which are assumed to be specifically characteristic for our evolution problem. The superior stability allows bigger timesteps independent of the spatial grid size. For a given uniform grid and given initial data we successively incremented the timestep size $\tau$ until oscillations occurred. The tables below show this experimental 'maximal' timestep size $\tau_{\max }$ for different grids and initial values. It turns out that in the relevant range the maximal timestep size is independent of the spatial grid size $h$.

TABLE 1

Maximal timesteps $\tau_{\max }$ for different initial data and different grid size $h$ on a domain $\Omega=(0,1)^{2}\left(\chi_{x<1 / 2}\right.$ denotes the characteristic function of the subdomain $(0,0.5) \times(0,1), b=0.4)$

\begin{tabular}{|c|c|}
\hline \multicolumn{2}{|c|}{ Initial data: $-b+2 b \chi_{x<1 / 2}$} \\
\hline$h$ & $\tau_{\max }$ \\
\hline \hline $1 / 16$ & 0.0235 \\
\hline$\sqrt{2} / 32$ & 0.0260 \\
\hline $1 / 32$ & 0.0210 \\
\hline$\sqrt{2} / 64$ & 0.0200 \\
\hline
\end{tabular}

\begin{tabular}{|c|c|}
\hline \multicolumn{2}{|c|}{ Initial data: $-b+2 b x$} \\
\hline$h$ & $\tau_{\max }$ \\
\hline \hline $1 / 16$ & 0.0185 \\
\hline$\sqrt{2} / 32$ & 0.0190 \\
\hline $1 / 32$ & 0.0180 \\
\hline$\sqrt{2} / 64$ & 0.0175 \\
\hline
\end{tabular}

\begin{tabular}{|c|c|}
\hline \multicolumn{2}{|c|}{ Random initial data } \\
\hline$h$ & $\tau_{\max }$ \\
\hline \hline $1 / 16$ & 0.0190 \\
\hline$\sqrt{2} / 32$ & 0.0185 \\
\hline $1 / 32$ & 0.0190 \\
\hline$\sqrt{2} / 64$ & 0.0190 \\
\hline
\end{tabular}

Together with the adaptive grid refinement strategy, the $\Theta$-scheme allows us to perform calculations of complex configurations in reasonable time. Figure 3, for example, shows an interesting effect of temporary negative coarsening. Even without elasticity, a long, bar-shaped particle splits up into several small, ball-shaped particles. After that, the usual coarsening of the particles takes place.

\section{Implementational aspects and adaptive grids}

We implemented the numerical schemes described above on a 2D triangular grid, generated by successive refinement of some prescribed macro-triangulation.

After assembling the matrices, any timestep requires the solution of several large systems of algebraic equations. In the first and third steps of the $\theta$-splitting scheme the systems are linear and symmetric. Hence we used the conjugate gradient method with a BPX preconditioning to solve them. However, the second step is nonlinear and so we applied Newton's method. The resulting linear systems are not symmetric and therefore a GMRES algorithm [26] was applied in each Newton iteration.

For efficiency, the calculations were based on an adaptively refined grid. After each timestep, local error indicators were calculated heuristically. These error indicators then governed the refinement and coarsening process of the grid. The heuristic strategy was to refine the transition zones while coarsening the phases.

To identify the transition zone several approaches proved to be equally suitable. One could either refine regions where the norm of the gradient of the solution is bigger than a certain threshold, or 

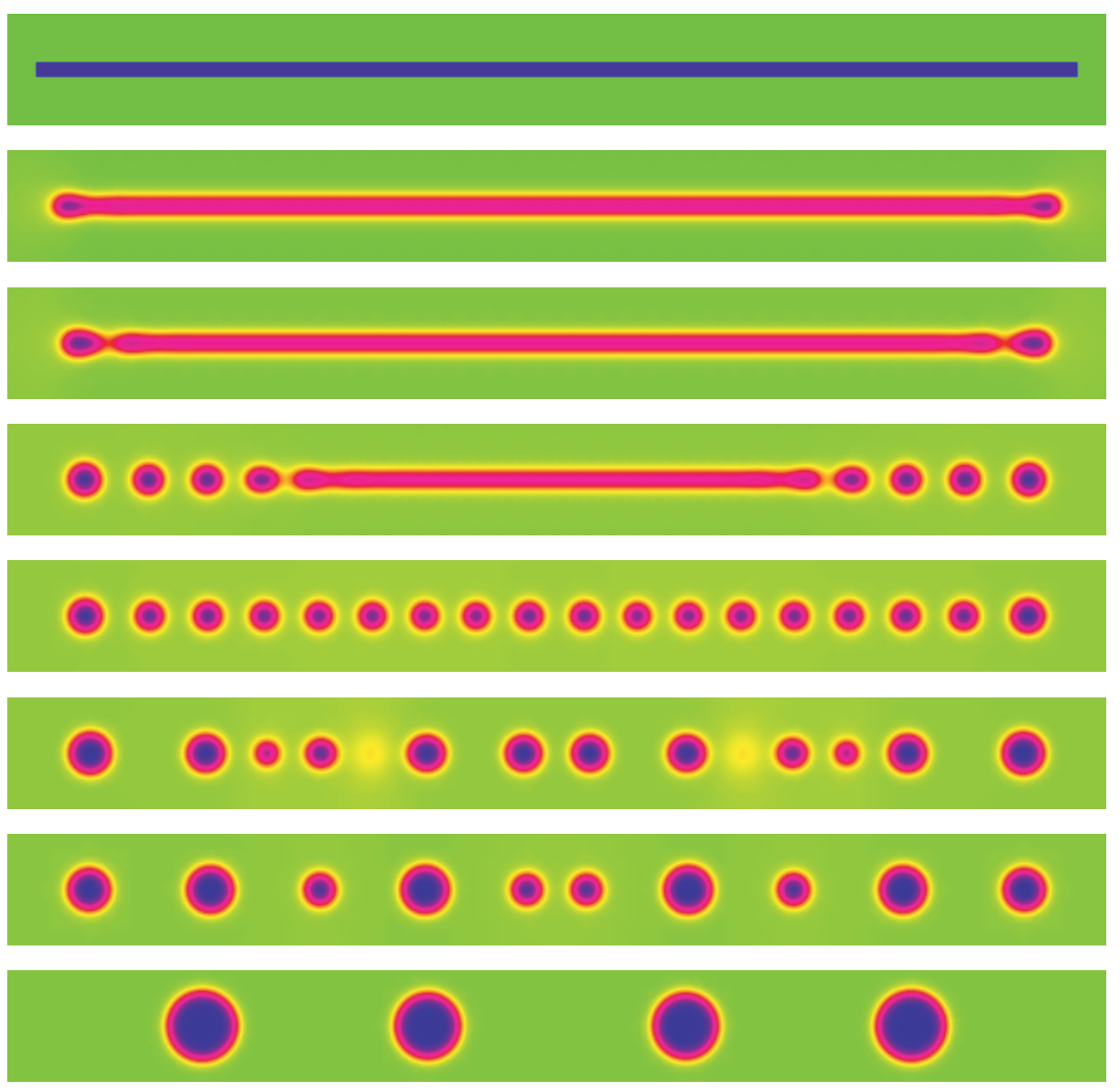

FIG. 3. Deformation of a bar-shaped particle (without elasticity; initial value, $t=0.01, t=0.02, t=0.05, t=0.1, t=0.2$, $t=0.5$ and $t=2.0$ ).

where the concentration is far from the minima of the nonlinearity $\psi$. Figure 4 shows the adaptive grid of two timesteps of the evolution shown in Fig. 3.

\section{Numerical results}

In all computational experiments we used the function $\psi$ of (1.1) which has a double well form and the constant $b$ was chosen to be 0.4 . The gradient energy coefficient $\gamma$ in the Cahn-Hilliard equation was set to be $10^{-5}$, although in our implementation smaller values of $\gamma$ can also be handled.

Figure 5 demonstrates the impact of elasticity in the Cahn-Hilliard model. In both calculations the initial value has been one homogeneous phase with a small random perturbation. Whereas in the case without elasticity, ball-shaped areas evolve after the phase separation has taken place, cubic anisotropic elasticity causes a more rectangular shape of the particles. For the elasticity tensor we 


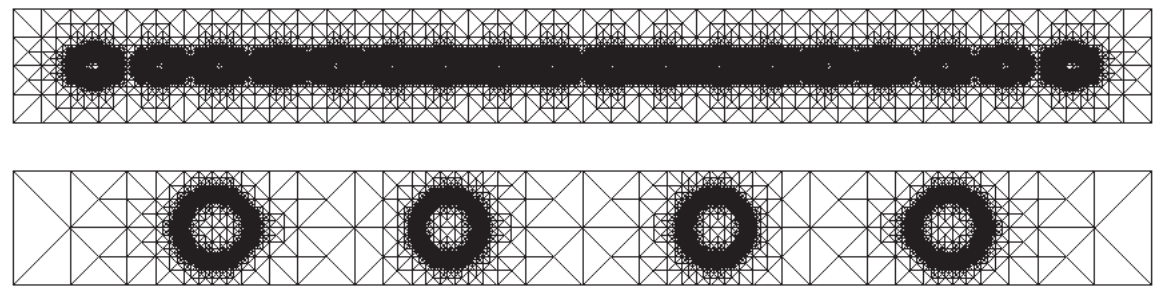

FIG. 4. Adaptive grids corresponding to the evolution in Fig. 3.

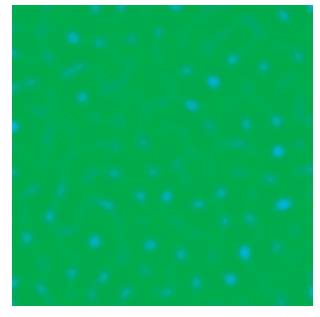

(a)

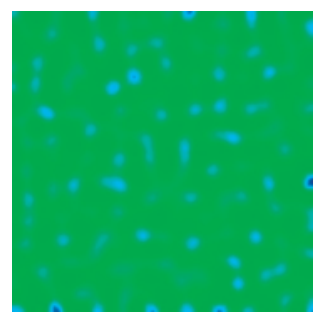

(e)

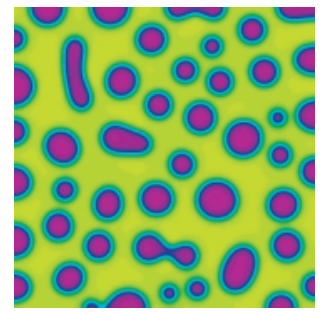

(b)

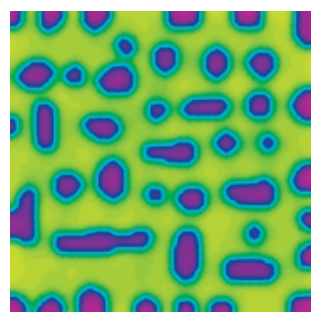

(f)

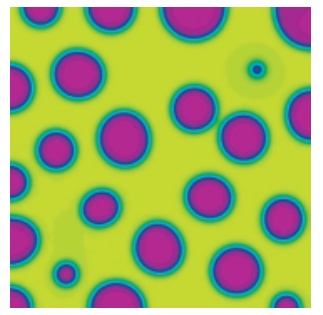

(c)

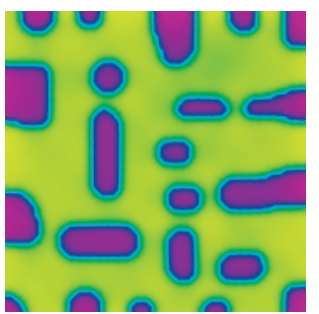

(g)

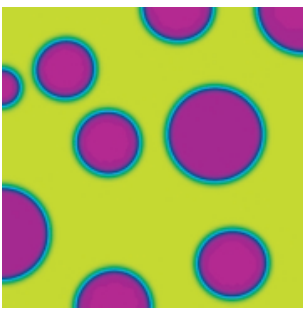

(d)

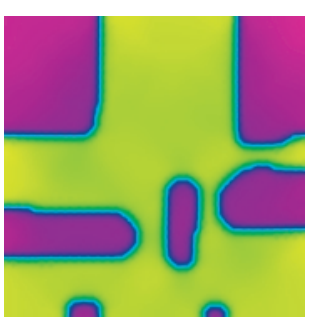

(h)

FIG. 5. Evolution without (top) and with (bottom) elasticity. (a) $t=0.01$, (b) $t=0.2$, (c) $t=1.0$, (d) $t=7.5$, (e) $t=0.01$, (f) $t=0.2$, (g) $t=1.0$, (h) $t=7.5$.

chose

$$
C_{1111}=2 \quad C_{1122}=1 \quad C_{1212}=10 .
$$

The solution of the elastic system is illustrated in Fig. 6. Starting from a ball-shaped initial value the anisotropy can at once be seen in the trace of the strain tensor. The corresponding displacement field is presented in the right Fig. 6 where the deformation $\Phi=x+25 \boldsymbol{u}(x)$ is 'applied' to a chequer pattern.

Figure 7 shows the case where $\gamma=0$. As initial value we chose the evolution shown in the top row of Fig. 5 at the time $t=1.0$. In this simulation $\gamma$ was $10^{-5}$ and the elasticity was switched off. Setting $\gamma=0$ leads even for small timesteps to oscillations that immediately rendered the results meaningless. The oscillation patterns after a small single timestep are shown in the middle of Fig. 7. 

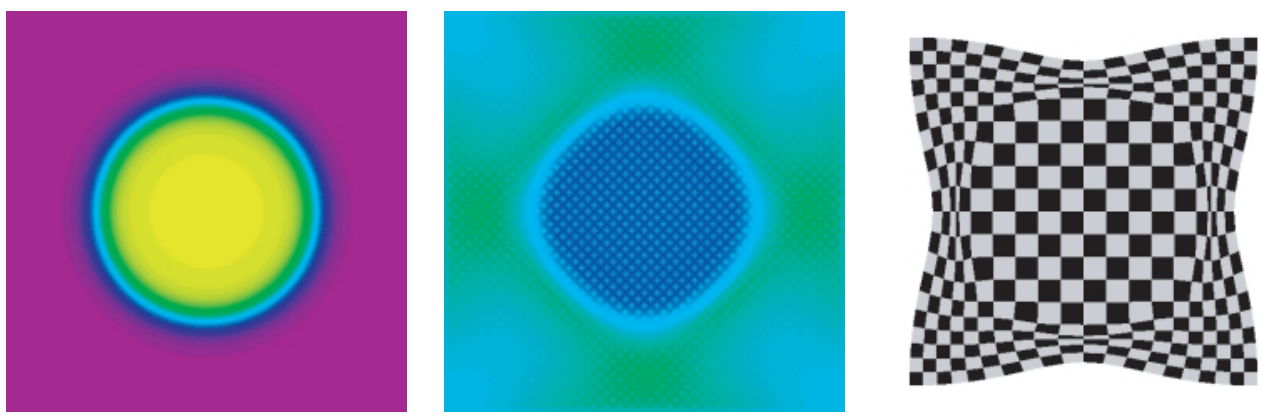

FIG. 6. Initial value, $\operatorname{tr} \mathcal{S}$ with positive anisotropic elasticity and (strongly scaled) displacement field.
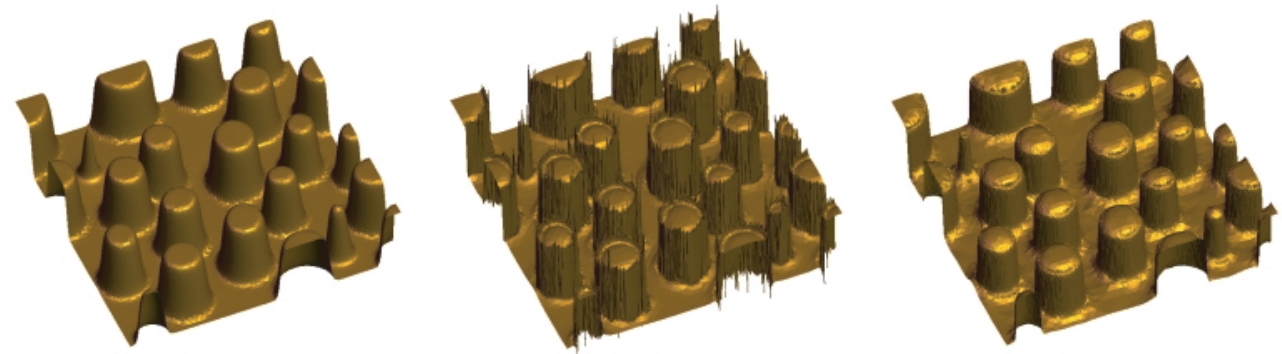

FIG. 7. The case $\gamma=0$ : Initial value, without elasticity and with elasticity.

Starting again from the initial value now with elastic effects present leads to stable solutions with sharp interfaces as depicted in the right part of Fig. 7.

Finally, we present some calculations regarding the error estimates proven above. As there are no analytic solutions for the Cahn-Hilliard equation known, it is difficult to devise a sensible test situation in which a meaningful experimental error can be computed. We proceed as follows. We prescribe initial data $\rho_{0}=b \cdot \tanh (10 x)$ where $b=0.4$ is the constant from (1.1). Using this initial data we calculate a reference solution applying 512 timesteps of timestep size $\tau=10^{-5}$ on a uniform grid with grid size $h=2^{-8}$. First we compare the result of the final timestep to numerical solutions on coarser but still uniform grids. Table 2 shows the difference between the reference solution and respective solutions on coarser grids. These are measured in the $L_{2^{-}}$and in the $L_{\infty^{-}}$ norm. We divided the timestep size by four for any halfening of the grid size. The results give a strong indication for the predicted second-order convergence.

To give experimental evidence for the applicability of adaptive grids to the problem at hand we compare the reference solution with a series of solutions on adaptive grids. Starting from an adaptive resolution of the initial data with a minimum element size of $h_{\min }=2^{-4}$ we generate successively finer adaptive grids by global refinement up to an minimal element size of $h_{\min }=2^{-7}$. On each grid we calculate a numerical solution. For the adaptive approximation of the initial data we apply the gradient-based marking strategy already mentioned in Section 5. Again, the expected second-order convergence now with respect to the finest gridsize can be seen. 
TABLE 2

Evaluation of the experimental numerical error for a test example.

\begin{tabular}{|c|c|c|c|c|}
\hline \multicolumn{5}{|c|}{ uniform grid } \\
\hline$h$ & $\left\|\rho_{h}-\rho_{\text {ref }}\right\|_{L^{\infty}(\Omega)}$ & $\left\|\rho_{h}-\rho_{\text {ref }}\right\|_{L^{2}(\Omega)}$ & $\left\|\boldsymbol{u}_{h}-\boldsymbol{u}_{\text {ref }}\right\|_{L^{\infty}(\Omega)}$ & $\left\|\boldsymbol{u}_{h}-\boldsymbol{u}_{\text {ref }}\right\|_{L^{2}(\Omega)}$ \\
\hline \hline $2^{-4}$ & $4.620019 \mathrm{e}-02$ & $1.103694 \mathrm{e}-02$ & $1.067519 \mathrm{e}-03$ & $1.131053 \mathrm{e}-04$ \\
\hline $2^{-5}$ & $1.609030 \mathrm{e}-02$ & $2.728970 \mathrm{e}-03$ & $3.615097 \mathrm{e}-04$ & $2.767725 \mathrm{e}-05$ \\
\hline $2^{-6}$ & $3.909958 \mathrm{e}-03$ & $6.675369 \mathrm{e}-04$ & $8.771903 \mathrm{e}-05$ & $6.330371 \mathrm{e}-06$ \\
\hline $2^{-7}$ & $2.104562 \mathrm{e}-03$ & $3.521095 \mathrm{e}-04$ & $4.132785 \mathrm{e}-05$ & $3.630578 \mathrm{e}-06$ \\
\hline
\end{tabular}

\begin{tabular}{|c|c|c|c|c|}
\hline \multicolumn{5}{|c|}{ adaptively refined grid } \\
\hline$h_{\min }$ & $\left\|\rho_{h}-\rho_{\text {ref }}\right\|_{L^{\infty}(\Omega)}$ & $\left\|\rho_{h}-\rho_{\text {ref }}\right\|_{L^{2}(\Omega)}$ & $\left\|\boldsymbol{u}_{h}-\boldsymbol{u}_{\text {ref }}\right\|_{L^{\infty}(\Omega)}$ & $\left\|\boldsymbol{u}_{h}-\boldsymbol{u}_{\text {ref }}\right\|_{L^{2}(\Omega)}$ \\
\hline \hline $2^{-4}$ & $4.612586 \mathrm{e}-02$ & $1.121934 \mathrm{e}-02$ & $1.337114 \mathrm{e}-03$ & $2.556929 \mathrm{e}-04$ \\
\hline $2^{-5}$ & $1.594744 \mathrm{e}-02$ & $2.888784 \mathrm{e}-03$ & $4.358494 \mathrm{e}-04$ & $6.459105 \mathrm{e}-05$ \\
\hline $2^{-6}$ & $4.199800 \mathrm{e}-03$ & $7.404723 \mathrm{e}-04$ & $1.157450 \mathrm{e}-04$ & $1.397157 \mathrm{e}-05$ \\
\hline $2^{-7}$ & $6.636309 \mathrm{e}-04$ & $1.468596 \mathrm{e}-04$ & $1.647358 \mathrm{e}-05$ & $2.742585 \mathrm{e}-06$ \\
\hline
\end{tabular}

\section{REFERENCES}

1. Barett, J. W. \& Blowey, J. F. An error bound for the finite element approximation of the CahnHilliard equation with logarithmic free energy. Numer. Math. 72, (1995) 1-20.

2. BARETT, J. W. \& Blowey, J. F. An improved error bound for the finite element approximation of a model for phase separation of a multi-component alloy. IMA J. Numer. Anal. 19, (1999) 147-168.

3. Bristeau, M. O., Glowinski, R., \& PeriauX, J. Numerical methods for the Navier-Stokes equations: applications to the simulation of compressible and incompressible viscous flows. In Computer Physics Report UH/MD-4. University of Houston, (1987).

4. CAHN, J. W. On spinodal decomposition. Acta Metall. 9, (1961) 795-801.

5. CAhn, J. W. \& Hilliard, J. E. Free energy of a non-uniform system i. interfacial free energy. J. Chem. Phys. 28, (1958) 258-267.

6. Carrive, M., Miranville, A., \& PiÉtrus, A. The Cahn-Hilliard equation for deformable elastic continua. Adv. Math. Sci. Appl. to appear.

7. Ciarlet, P. G. The Finite Element Method for Elliptic Problems. North-Holland, Amsterdam (1978).

8. Copetti, M. I. M. \& Elliott, C. M. Numerical analysis of the Cahn-Hilliard equation with logarithmic free energy. Numer. Math. 63, (1992) 39-65.

9. DReYeR, M. \& MÜLler, W. H. A study of the coarsening in tin/lead solders. WIAS Preprint Berlin (1998) 444.

10. Quiang Du, \& Nicolaides, R. A. Numerical analysis of a continuum model of phase transition. SIAM J. Numer. Anal. 28, (1991) 1310-1322.

11. Elliott, C. M. The Cahn-Hilliard model for the kinetics of phase separation. In: Rodrigues, J. F. (ed), In Mathematical Models for Phase Change Problems, International Series of Numerical Mathematics, vol. 88. pp. 35-73. Birkhäuser, Basel (1989).

12. Elliott, C. M., French, D. A., \& Milner, F. A. A 2nd order splitting method for the Cahn-Hilliardequation. Numer. Math. 54, (1989) 575-590.

13. Elliott, C. M. \& LaRsSon, S. Error estimates with smooth and nonsmooth data for a finite element method for the Cahn-Hilliard equation. Math. Comput. 58, (1992) 603-630.

14. Eshelby, J. D. Elastic inclusions and inhomogeneities. Prog. Solid Mech. 2, (1961) 89-140.

15. Fratzl, A., Penrose, O., \& Lebowitz, J. L. Modelling of phase separation in alloys with coherent elastic misfit. J. Stat. Phys. 95, (1999) 1429-1503. 
16. French, D. A. \& Schaeffer, J. W. Continuous finite element methods which preserve energy properties for nonlinear problems. Appl. Math. Comput. 39, (1990) 271-295.

17. GARCKE, H. On mathematical models for phase separation in elastically stressed solids, Habilitation, $\mathrm{PhD}$. Thesis, University Bonn, (2000).

18. GitT, A. F. Modellierung der Phasenumwandlung einer Al-Cu-Legierung mit hilfe einer numerischen Lösung der Cahn-Hilliard-Gleichung, Diploma Thesis, RWTH Aachen, (1997).

19. Khachaturyan, A. G. Some questions concerning the theory of phase transitions in solids. Fiz. Tverd. Tela. 8, (1966) 2709-2717. (English translation in Sov. Phys. Solid State 8 (1966), 2163.)

20. LeO, P. H., Lowengrub, J. S., \& JoU, H. J. A diffuse interface model for microstructural evolution in elastically stressed solids. Acta Mater. 46, (1998) 2113-2130.

21. ModicA, L. The gradient theory of phase transition and the minimal interface criterion. Arch. Rat. Mech. Anal. 98, (1987) 123-142.

22. MÜller-UrbaniaK, S. Eine Analyse des Zweischritt- $\theta$-Verfahrens zur Lösung der instationären Navier-Stokes-Gleichungen. Preprint des SFB 359 (1994) 94-01.

23. Novick-Cohen, A. The Cahn-Hilliard equation: mathematical and modelling perspectives. Adv. Math. Sci. Appl. 8, (1998) 965-985.

24. Strang, G. On the construction and comparison of difference schemes. SIAM J. Numer. Anal. 5, (1968) 506-517.

25. Thomée, V. Galerkin Finite Element Methods for Parabolic Problems. Springer, (1984).

26. WALKER, H. F. Implementation of the gmres method using householder transformations. SIAM J. Sci. Stat. Comput. 9, (1988) 152-163.

27. WeIKARD, U. Numerische lösungen der Cahn-Hilliard-Gleichung unter einbeziehung elastischer materialeigenschaften, Diploma Thesis, University Bonn, (1998). 